\title{
Colorectal Cancer Cell Line SW480 and SW620 Released Extravascular Vesicles: Focus on Hypoxia-induced Surface Proteome Changes
}

\author{
ILVA NAKURTE ${ }^{1}$, KASPARS JEKABSONS ${ }^{2}$, REINIS REMBERGS ${ }^{2}$, ELINA ZANDBERGA ${ }^{3}$, \\ ARTURS ABOLS ${ }^{3}$, AIJA LINE $\bar{E}^{3}$ and RUTA MUCENIECE ${ }^{2}$ \\ ${ }^{1}$ Department of Physical Chemistry, Faculty of Chemistry, University of Latvia, Riga, Latvia; \\ ${ }^{2}$ Department of Pharmacy, Faculty of Medicine, University of Latvia, Riga, Latvia; \\ ${ }^{3}$ Latvian Biomedical Research and Study Centre, Riga, Latvia
}

\begin{abstract}
Background/Aim: Extravascular vesicle (EV) proteome closely reflects the proteome of the cell of origin. Therefore, cancer cell-derived EV proteomic analysis could help in identifying cancer biomarkers. This study's goal was to investigate hypoxia-induced proteomic changes in EV released from hypoxic human isogenic non-metastatic colorectal cancer cells SW480 and metastatic colorectal cancer cells SW620. Materials and Methods: EV were characterized by western blot, transmission electron microscopy, proteomic analysis using liquid chromatography time-of-flight-mass spectrometry and quantified by an labelfree intensity-based absolute quantitation ( $i B A Q)$ approach. Results: A total of 16 proteins in hypoxic EV exceeded normoxic EV protein levels in SW480 EV. Of them, 15 were also found in EV of hypoxic SW620 cells. The expression levels of proteins differed quantitatively: iBAQ (log 10) scores of the levels of five proteins in SW620 EV exceeded those in hypoxic SW480 EV and levels of 11 proteins in SW480 EV exceeded those of SW620 EV. Conclusion: Under hypoxia, colorectal cancer cells release EV that qualitatively and quantitatively change the surface proteome. In the future, the specific hypoxia-induced proteins could be developed as new biomarkers for non-invasive assessment of tumour hypoxia.
\end{abstract}

Correspondence to: Ruta Muceniece, Department of Pharmacy, Faculty of Medicine, University of Latvia, Str. Jelgavas 1, Riga LV1004, Latvia. Tel: +371 26437377, e-mail: ruta.muceniece@lu.lv and Ilva Nakurte, Department of Physical Chemistry, Faculty of Chemistry, University of Latvia, Str. Jelgavas 1, Riga LV-1004, Latvia. Tel: +371 26330172, e-mail: ilva.nakurte@gmail.com

Key Words: Colorectal cancer cell lines SW480 and SW620, extravascular vesicles, hypoxia, proteome, label-free protein quantification.
Nowadays early cancer diagnosis and public awareness of risk factors for cancer have significantly improved patients' survival. However, there is still a need for more specific cancer markers, which could support earlier diagnosis and help choose more precisely therapy for each individual patient. Recently, tumour cell-derived extravascular vesicles (EV), including exosomes (Ex) and microvesicles have been considered as a source for the discovery of relevant biomarkers for cancer diagnosis. However, numerous types of cells secrete EV into the extracellular environment (1-3). EV have also been identified in most of the bodily fluids. Therefore, it is important to find out tumour cell-specific features to differentiate tumor cell released EV from those released from other cells. Recently, studies focused on searching for specific biomarkers in different cancer cell line-released EV, tumour tissues, as well as in EV found in bodily fluids, mainly blood and urine (4-6). Over the last decade, it has been revealed that EV contain a complex composition of molecules, including proteins, lipids, miRNA and mRNA. Previous studies on exosome biomarker application in cancer diagnosis have been focused on miRNAs $(4,7,8)$, whereas surface protein profiling of EV have been done using antibody microarrays $(6,7)$ or studied via biochemical assays and mass spectrometry with emphasis on mass-spectrometry-based "omic" analytical techniques (9). Currently, our understanding of EV contents is confined by the limited cells from which vesicles have been characterized (10, 11). In our previous study, using ultra-high-performance liquid chromatography (UHPLC) time of flight (TOF) mass spectrometry (MS) method, we have demonstrated specific hypoxia-induced proteome changes in SW480 cell released EV surface proteome in comparison with normoxic cell EV (12). The hypoxia model was chosen because hypoxia is one of the microenvironment factors known to induce genetic and proteomic changes in cancer cells (13).

The goal of the current study was to find out whether there are detectable surface proteome changes in exosome- 
enriched EV released from hypoxic human isogenic nonmetastatic colorectal cancer cells SW480 and metastatic colorectal cancer cells SW620 in comparison with those released from the cells grown in normoxic conditions. By using label-free intensity-based absolute quantitation (iBAQ) approach we also analyzed the quantity of the discovered proteins. We suggest that hypoxia initiated EV surface proteome changes might highlight proteins which in the future could serve as hint in cancer biomarker discovery.

\section{Materials and Methods}

SW480 and SW620 cell culture. The human colorectal adenocarcinoma cell lines SW480 and SW620 were purchased from the American Type Culture Collection (ATCC, Manassas, VA, USA). Cells were cultured in DMEM/F12 medium (Lonza, Basel, Switzerland \#BE12-79F) supplemented with $10 \%$ foetal bovine serum (Sigma- Aldrich, St. Louis, MO, USA, \#F7524, 1\% Lglutamine (Lonza, \#17-605E) and 1\% antibiotic-antimycotic (Gibco, Thermo Fisher Scientific, Waltham, MA, USA, \#15240-062) in a humidified $5 \% \mathrm{CO}_{2}$ atmosphere at $37^{\circ} \mathrm{C}$. The cell cultures were monitored by mycoplasma infection by using PCR Mycoplasma Test Kit I/C (PromoKine, PromoCell GmbH, Heidelberg, Germany, \# PK-A91-1096). For the isolation of EV, $1 \times 10^{6}$ cells per $\mathrm{ml}$ were seeded in serum -free DMEM/F12 medium supplemented with $1 \%$ L-glutamine (Lonza, \#BE12-605E), $10 \mathrm{ng} / \mathrm{ml}$ beta fibroblast growth factor (bFGF) (Santa Cruz, Biotechnology, Dallas, TX, USA, \#sc4573), $20 \mathrm{ng} / \mathrm{ml}$ epidermal growth factor (EGF) (R\&D Systems, Minneapolis, MN, USA, \#236-EG-200), $50 \mathrm{ng} / \mathrm{ml}$ hydrocortisone (Sigma-Aldrich, H0135), 1×B27 (Invitrogen, Fisher Scientific, Leicestershire, UK \#17504001) and grown for $48 \mathrm{~h}$ in suspension at hypoxic $\left(1 \%\right.$ oxygen, $94 \%$ nitrogen, $\left.5 \% \mathrm{CO}_{2}\right)$ or normoxic $(20 \%$ oxygen, $75 \%$ nitrogen, $5 \% \mathrm{CO}_{2}$ ) conditions.

EV isolation. Culture media were collected and centrifuged at $300 \times \mathrm{g}$ for $5 \mathrm{~min}$ to remove the cells and at $3,000 \times \mathrm{g}$ for $30 \mathrm{~min}$ at $+4^{\circ} \mathrm{C}$ to remove cell debris, and then filtered through $0.2 \mu \mathrm{m}$ filters (Sarstedt, Numbrecht, Germany, \#83.1826.001). After filtration, media were concentrated up to $1 \mathrm{ml}$ with $100 \mathrm{kDa}$ centrifuge filters (Merck Millipore, MA, USA \#UFC910024). Size exclusion chromatography (SEC) was performed to separate EV from protein aggregates by using CL6B sepharose (GE healthcare, Buckinghamshire, UK \#17-0160- 01) filled columns (Kinesis, 9000000-016T). Further, each SEC fraction was measured by Zetasizer Nano ZS and all fractions containing particles ranging in size from 30 to $200 \mathrm{~nm}$ were pooled and concentrated up to $100 \mu \mathrm{l}$ by $3-\mathrm{kDa}$ filters (Merck Millipore, UFC500324) and aliquoted to avoid repeated freeze/thaw cycles. Isolated EV where characterized by western blot (WB) and Transmission Electron Microscopy (TEM).

Immunofluorescence. Multicellular SW480 and SW620 spheroids were centrifuged at $300 \times g$ for 5 min onto slides coated with polyL-lysine, fixed with cold methanol/acetone for $20 \mathrm{~min}$ at $-20^{\circ} \mathrm{C}$, blocked with $2 \%$ bovine serum albumin (BSA) in phosphatebuffered saline (PBS) for $30 \mathrm{~min}$ at room temperature. Then the slides were incubated with primary antibodies - rabbit anti- CAIX (Abcam, Cambridge, UK, \#ab15086) diluted (1:50) and mouse monoclonal anti-HIF1 $\alpha$ (R\&D Systems, MAB1935) diluted (1:50) overnight at $+4^{\circ} \mathrm{C}$. After incubation with the primary antibodies, slides were washed and incubated for $1 \mathrm{~h}$ at room temperature with secondary antibodies - goat anti-rabbit IgG conjugated with fluorescein (FITC) (Jackson ImmunoResearch, Cambridgeshire, UK, \#11-095-006) or goat anti-mouse IgG conjugated with cyanine (Cy3) (Jackson ImmunoResearch, 115-165-0) diluted (1:200) and mounted with ProLong Gold Antifade Mountant with DAPI (Thermo Fisher Scientific, \#P36935).

Western Blot $(W B)$. Proteins were isolated from EV with RIPA buffer. One microgram of EV total protein was loaded per lane and separated by $10 \%$ SDS-PAGE. Proteins were electro blotted onto nitrocellulose membranes and blocked with $10 \%$ (w/v) fat-free milk and then incubated with the primary antibodies: CAIX (Abcam, ab15086) (1:1,000), ALIX (Santa Cruz, sc-166952) (1:250), TSG101 (Abcam, ab125011) (1:1,000), Calnexin (Abcam, ab22595) (1:1000) and $\beta$-actin (Abcam, ab8224) (1:2000). After washing, the membranes were incubated with peroxidase - conjugated rabbit antimouse secondary antibody (Santa Cruz, sc-516102) (1:2000) or mouse anti-rabbit secondary antibody (Santa Cruz, sc-2357) (1:2000). Next, the membranes were washed and processed with ECL select western blotting detection reagents (GE Healthcare, RP2235) according to the manufacturer's instructions.

Proteomics of EVs. The concentration of EV proteins was measured using a Nano Drop and Pierce BCA Protein assay kit (ThermoFisher Scientific, Wilmington, DE, USA) and normalized as starting input material to the protein concentration $1 \mathrm{mg} / \mathrm{ml}$. EV surface protein fraction was separated using XPEP Exosome Mass Spec kit following manufacturer's instructions (System Biosciences, Palo Alto, CA, USA, \# XPEP100A1). The XPEP1000A-1 reagent kit comes complete to create EV surface protein "shaving" peptide libraries or complete EV peptide libraries. We have used the protocol for XPEP-Shave surface protein libraries. At the end of the protocol, EV peptide libraries eluted from $10 \mathrm{kD}$ cut-off spin columns for Mass Spec sample preparation in the collection tube were $50 \mu \mathrm{l}$ in volume with approximately $0.4 \mu \mathrm{g}$ protein concentration (XPEP-Shave). According to the manufacturer's instructions, peptide libraries were ready to load on mass spectrometer directly. Chromatographic analyses were performed on a modular UHPLC system (Agilent 1290 Infinity series). Liquid chromatography separations were achieved by using an Extend-C18 RRHD column $(2.1 \times 50 \mathrm{~mm}, 1.8 \mu \mathrm{m})$. Elution solvents consisted of $0.1 \%$ formic acid in acetonitrile and $0.1 \%$ formic acid in water in gradient mode at a flow rate of $0.3 \mathrm{ml} / \mathrm{min}$. The high-resolution mass spectra were taken on an Agilent 6230 TOF LC/MS with electrospray ionization in positive mode. Mass spectra were collected over an m/z range of 300-2,500 Da. Protein structures corresponding to mass spectra were searched in UNIPROT-SPROT + UNIPROT-TREMBL and ExoCarta data basis with taxonomy Homo sapiens (human), enzymatic cleavage=trypsin, fixed modifications $=$ carbamidomethyl $(\mathrm{C})$, variable modifications $=$ oxidation $(\mathrm{M})$, monoisotopic mass, peptide tolerance $=1.5 \mathrm{Da}, \mathrm{MS}$ tolerance $=0.8 \mathrm{Da}$, one missed cleavage, charge positions $=1+$, and $2+$, instrument=ESI-QTOF. Relative protein intensity was calculated in intensity based absolute quantification scores - iBAQ $(\log 10)$ and was used for label-free quantification of particular protein (14). The obtained data from the UHPLC-TOF-MS runs were processed using Agilent MassHunter BioConfirm Software. Obtained sequences were used to search for full length proteins using standard human proteome database UniProt and MaxQuant 
A

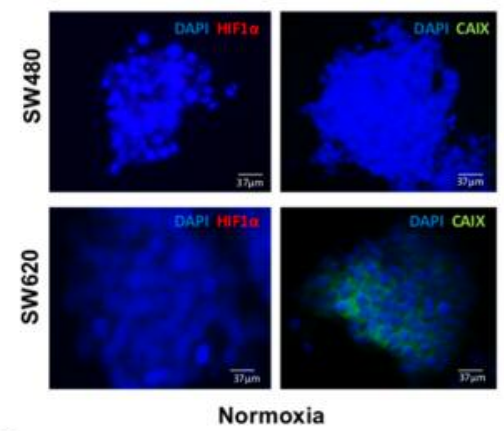

C

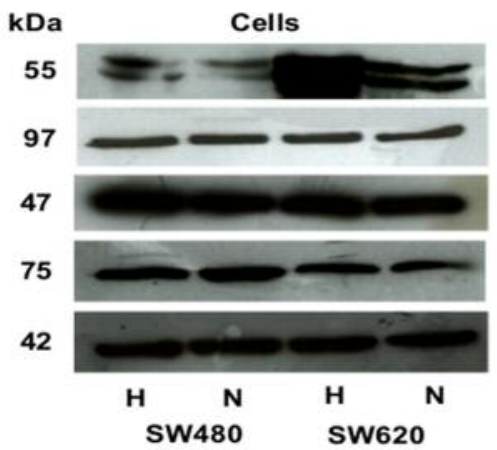

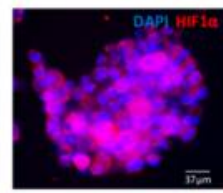
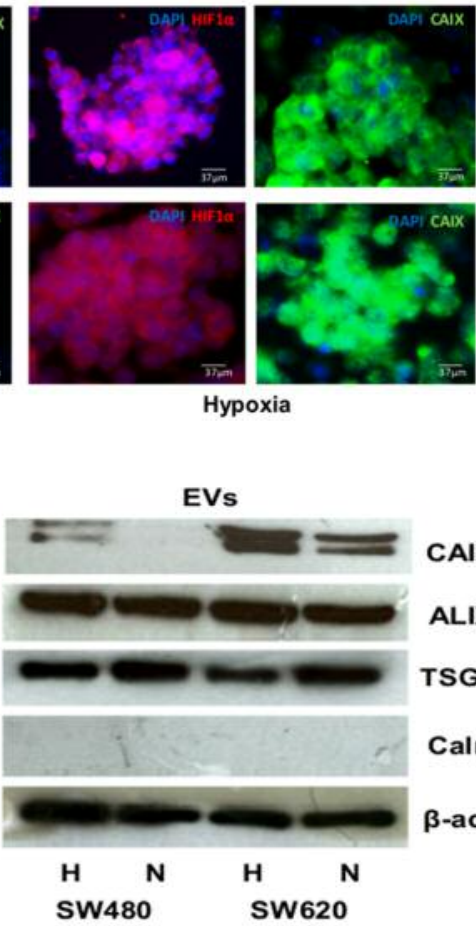

B

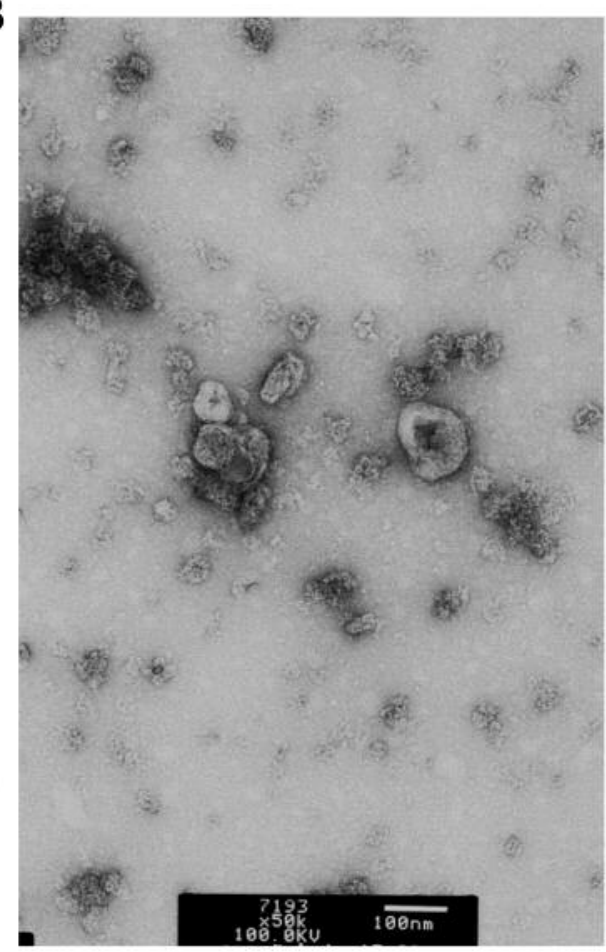

Figure 1. Isolation and characterization of SW480 and SW620 EVs. A) Immunofluorescence analysis of SW480 and SW620 multicellular spheroids

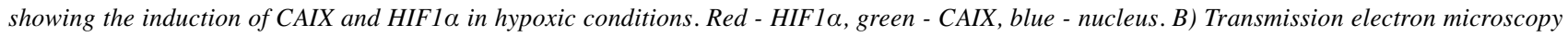
of EVs showing a cup shaped morphology typical to exosomes. White scale bar represents $100 \mathrm{~nm} . \mathrm{C}$ ) Western blot analysis of EVs markers (CAIX, ALIX, TSG101) and negative control (Calnexin) in EVs. H: Hypoxia; N: normoxia.

software. To confirm that the found proteins are Ex proteins, we included a search in ExoCarta data basis. Protein subcellular localization data were obtained from the UniProt database.

\section{Results}

Verification of hypoxic conditions and characterization of EV. SW480 and SW620 cells were cultured in hypoxic and normoxic conditions for $48 \mathrm{~h}$. The hypoxic conditions were verified by immunofluorescence with antibodies against HIF1 $\alpha$ and CAIX that are well known markers of hypoxia (Figure 1A). EV were isolated from media by SEC and characterized by TEM and western blot analysis. TEM revealed that the particles in EVs samples ranged in size from 30 to $100 \mathrm{~nm}$ and had a typical cup-shaped morphology characteristic of Ex (Figure 1B). Western blot analysis showed that the EV were positive for classical EVs markers ALIX and TSG101, and negative for the endoplasmic reticulum protein calnexin, thus showing that the EV samples were not contaminated with cellular components (Figure 1C). Taken together, these results showed that the obtained small size fraction of EV is enriched with Ex.
Protein analysis. LC-MS data showed distinct differences between chromatograms obtained from $\mathrm{EV}^{\mathrm{Hyp}}$ surface protein libraries and those from normoxic $\mathrm{EV}^{\text {Norm }}$. Further, we searched for differences between $\mathrm{EV}^{\mathrm{Norm}}$ and $\mathrm{EV}^{\mathrm{Hyp}}$ proteins by analyzing chromatograms and mass spectra of abundant $\mathrm{EV}^{\mathrm{Hyp}}$ proteins, without considering overlapping proteins unless there were obvious differences in their quantities. To obtain data on specific hypoxia-induced proteins, data were filtered to maintain only proteins that were more than three iBAQ $(\log 10)$. After selection of the above three iBAQ (log 10) hypoxia-induced EV surface proteins, the same proteins were examined in normoxic EVs samples. Table I shows the hypoxia-induced genes, EV surface proteins, the number of amino acids in each protein and the molecular weight in $\mathrm{kDa}$. Some of the listed proteins may not be localized only in the cell membrane. However, after finding them in the surface proteome of EVs, their localization in the membrane was assumed. Table II shows relative quantities of $\mathrm{EV}^{\mathrm{Hyp}}$ proteins in comparison with those in $\mathrm{EV}^{\mathrm{Norm}}$, as well as protein quantities in EV released from SW480 in comparison to those released from SW620. 
Table I. Identified hypoxia-induced proteins.

\begin{tabular}{|c|c|c|c|c|}
\hline No & Name & Gene & Length (AA) & $\mathrm{kDa}$ \\
\hline 1 & Lysosome-associated membrane glycoprotein 1 & $L A M P 1$ & 417 & 44.883 \\
\hline 2 & Integrin alpha-6 & ITGA6 & 1130 & 126.606 \\
\hline 3 & Coatomer subunit beta & $C O P B 1$ & 953 & 107.143 \\
\hline 4 & Homeobox protein TGIF1 & $T G I F 1$ & 401 & 43.013 \\
\hline 5 & 4F2 cell-surface antigen heavy chain & $S L C 3 A 2$ & 630 & 67.994 \\
\hline 6 & Sodium/potassium transporting ATPase subunit alpha-1 & ATP1A1 & 1023 & 112.896 \\
\hline 7 & Choline transporter-like protein 4 & ATP1A1 & 710 & 79.254 \\
\hline 8 & $\mathrm{Na} / \mathrm{H}$ exchange regulatory cofactor NHE-RF1 & $S L C 9 A 3 R 1$ & 358 & 38.868 \\
\hline 9 & Desmoplakin & $D S P$ & 2871 & 331.774 \\
\hline 10 & Ras GTPase-activating-like protein IQGAP1 & $I Q G A P 1$ & 1657 & 189.252 \\
\hline 11 & Aspartate aminotransferase, mitochondrial & GOT2 & 430 & 47.518 \\
\hline 12 & Secretory carrier-associated membrane protein 3 & SCAMP3 & 347 & 38.287 \\
\hline 13 & Immunoglobulin superfamily member 8 IGSF8 & $I G S F 8$ & 613 & 65.034 \\
\hline 14 & Disintegrin and metalloproteinase domain-containing protein 10 & ADAM10 & 748 & 84.142 \\
\hline 15 & Disco-interacting protein 2 homolog B & $D I P 2 B$ & 1576 & 171.492 \\
\hline 16 & Pituitary tumour-transforming gene 1 protein-interacting protein & PTTG1IP & 180 & 20.324 \\
\hline
\end{tabular}

AA: Number of amino acids.

Quantitative evaluation of protein expression in $\mathrm{BBAQ}$ scores $(\log 10)$ revealed that six specific $\mathrm{EV}^{\mathrm{Hyp}}$ proteins had iBAQ scores higher than three while the same proteins had iBAQ scores lower than three in SW480 EV ${ }^{\text {Norm }}$ namely, lysosome-associated membrane glycoprotein 1 (LAMP1), coatomer subunit beta (COPB1), homeobox protein TGinteracting factor 1 (TGIF1), 4F2 cell-surface antigen heavy chain (SLC3A2), sodium/potassium transporting ATPase subunit alpha-1 (ATP1A1) and choline transporter-like protein 4 (SLC44A4). Among them, LAMP1 was found only in SW480 $\mathrm{EV}^{\mathrm{Hyp}}$ samples, whereas another five proteins were also detected in SW620 released EV. Integrin alpha-6 (ITGA6) and COPB1 were found in the SW620 $\mathrm{EV}^{\mathrm{Hyp}}$ proteome, but not in $\mathrm{SW} 620 \mathrm{EV}^{\mathrm{Norm}}$. COPB1 was detected only in $\mathrm{EV}^{\mathrm{Hyp}}$ proteomes. $\mathrm{iBAQ}$ scores above seven ( $\log 10)$ were found for desmoplakin (DSP) in SW480 and SW620 $\mathrm{EV}^{\mathrm{Hyp}}$ proteomes, whereas Ras GTPaseactivating-like protein (IQGAP1) had iBAQ expression scores above seven only in SW480 $\mathrm{EV}^{\mathrm{Hyp}}$ proteome. Five proteins SLC3A2, ATP1A1, SLC44A4, DIP2B and PTTG1IP quantitatively dominated in SW620 $\mathrm{EV}^{\mathrm{Hyp}}$, whereas 11 proteins LAMP1, ITGA6, COPB1, TGIF1, SLC9A3R1, DSP, IQGAP1, GOT2, SCAMP3, IGSF8 and ADAM10 in SW480 EV Hyp.

\section{Discussion}

It has been suggested that the molecular content of EV, at least partially, reflects that of parent cells. EV transfer a variety of lipids, proteins, mRNAs, non-coding and structural RNAs and DNA fragments to recipient cells, thereby influencing their physiological functions (15). Moreover, EV
Table II. Quantitative comparison of abundantly hypoxia-induced proteins of EVs.

\begin{tabular}{|c|c|c|c|c|c|}
\hline \multirow[b]{2}{*}{ No } & \multirow[b]{2}{*}{ Gene } & \multicolumn{4}{|c|}{ iBAQ $(\log 10)$} \\
\hline & & $\begin{array}{c}480 \\
\text { EV }^{\text {Hyp }}\end{array}$ & $\begin{array}{c}480 \\
\text { EV }^{\text {Norm }}\end{array}$ & $\begin{array}{c}620 \\
\mathrm{EV}^{\mathrm{Hyp}}\end{array}$ & $\begin{array}{c}620 \\
\mathrm{EV}^{\text {Norm }}\end{array}$ \\
\hline 1 & $L A M P 1$ & 4.17 & $<3$ & $<3$ & $<3$ \\
\hline 2 & ITGA6 & 4.11 & 3.27 & 3.56 & $<3$ \\
\hline 3 & СОРB1 953 & 4.78 & $<3$ & 4.48 & $<3$ \\
\hline 4 & TGIF1 & 4.23 & $<3$ & 3.82 & 3.14 \\
\hline 5 & $S L C 3 A 2$ & 4.59 & $<3$ & 4.76 & 3.32 \\
\hline 6 & ATP1A1 & 4.59 & $<3$ & 4.76 & 3.32 \\
\hline 7 & $A T P 1 A 1$ & 5.12 & $<3$ & 5.53 & 4.35 \\
\hline 8 & SLC9A3R1 & 6.05 & 5.58 & 5.68 & 5.17 \\
\hline 9 & $D S P$ & 7.40 & 5.57 & 7.21 & 6.77 \\
\hline 10 & $I Q G A P 1$ & 7.02 & 4.38 & 6.83 & 6.31 \\
\hline 11 & GOT2 & 5.59 & 4.28 & 4.33 & 4.35 \\
\hline 12 & SCAMP3 & 6.00 & 3.38 & 5.90 & 4.67 \\
\hline 13 & $I G S F 8$ & 6.04 & 5.11 & 5.84 & 5.08 \\
\hline 14 & ADAM10 & 5.59 & 4.28 & 4.33 & 4.35 \\
\hline 15 & $D I P 2 B$ & 5.86 & 4.97 & 5.96 & 5.46 \\
\hline 16 & PTTG1IP & 5.80 & 5.00 & 6.03 & 5.50 \\
\hline
\end{tabular}

480: SW480; 620: SW620.

derived from hypoxic colorectal cancer cells confer the metastatic phenotype to non-metastatic cells (16). Thus, EV play an important role as mediators of intercellular communication and can transfer their contents to both the neighbouring cells and distant cells.

This study aimed to gain a deeper understanding into the EV surface proteome. 
In our studies we have followed International Society's for Extracellular Vesicles (ISEV) issued minimal set of biochemical, biophysical and functional standards to isolate and characterize EV (17). Our preparation of EV was enriched in Ex since as expected were found positive for CAIX, ALIX and TSG101, known markers of Ex. Mass spectrometry has been utilized to analyze proteins of $\mathrm{EV}$, however, this method is less specific than antibody-based technologies (18) due to the complicated sample preparation. Previously, proteomic analysis revealed that EV contain a common set of membrane and cytosolic proteins, and they also contain distinct subsets of proteins that could be associated with cell type-specific functions (19). In another study it was found that EV from SW480 cells contained proteins, that are involved in cell adhesion, while SW620-exosomal proteins are in cancer progression, metastasis and multi-drug resistance (20). Using UHPLC-TOF-MS, we obtained new data regarding hypoxiainduced proteome changes in SW620 and SW480 cell lines with emphasis on proteins at the surface of $\mathrm{EV}$, and a quantitative evaluation of hypoxia-induced proteins. Six specific SW480 $\mathrm{EV}^{\mathrm{Hyp}}$ proteins were not found in detectable levels in SW480 EV ${ }^{\text {Norm }}$ and five of them were also detected in SW $620 \mathrm{EV}^{\mathrm{Hyp}}$ in quantities that exceeded those in SW 620 $\mathrm{EV}^{\text {Norm }}$. Interestingly, LAMP1 was identified only in SW480 $\mathrm{EV}^{\mathrm{Hyp}}$. Earlier, it was suggested that LAMP1, also known as lysosome-associated membrane glycoprotein 1, and CD107a (Cluster of Differentiation 107a) may play a role in tumour differentiation and metastasis (21). LAMP1 has been found on the cell surface of highly metastatic tumour cells, especially in metastatic colon cancer cells, suggesting a role for LAMP1 in metastasis (22). Two proteins, TGA6 and COPB1, which were found to be abundant in SW620 cell released $\mathrm{EV}^{\mathrm{Hyp}}$, were expressed at negligible quantities in SW620 EV Norm. Integrins are known as main tetraspannin protein partners, whereas coatomer subunits alpha and beta, have been found in human colon cancer cell line LIM1863 released EV (23) and U-87MG glioblastoma cell released EV (24). Homeobox protein TGIF1 was overexpressed in both cell line released $\mathrm{EV}^{\mathrm{Hyp}}$. However, TGIF1 dominated in SW480 $\mathrm{EV}^{\mathrm{Hyp}}$. It is known that TGIF1 is significantly upregulated in colorectal cancers, and its high expression predicts poor prognosis (25). Thus, identification of these proteins in colorectal cancer cells or other cancer cells is confirmed by several authors. The list of proteins in $\mathrm{EV}^{\mathrm{Hyp}}$ and $\mathrm{EV}^{\text {Norm }}$ surface proteome demonstrates a differential effect of hypoxia in these cell lines. SW480 cells were influenced more by hypoxia than SW620, and this was reflected by the appearance of hypoxia-induced proteins in EV.

In conclusion, our results indicated that hypoxia induced distinct cell line-dependent EVs proteome changes in isogenic metastatic and non-metastatic colorectal cancer cell lines SW620 and SW480. Further studies are welcome to confirm these results in other cells and in vivo settings, and expose the role of specific proteins that are highly expressed in hypoxic EVs. Overall, the present study provided new evidence that under hypoxic conditions, the changes in the behavior of colorectal cancer cells could be reflected on the surface proteome of EVs.

\section{Conflicts of Interest}

The Authors declare that they have no conflicts of interest regarding this work.

\section{Acknowledgements}

This work was funded by Latvian Science Council Grant No. Nr.625/2014.

\section{References}

1 Wu J, Qu Z, Fei ZW, Wu JH and Jiang CP: Role of stem cellderived exosomes in cancer. Oncol Lett 13: 2855-2866, 2017.

2 Ramteke A, Ting H, Agarwal C, Mateen S, Somasagara R, Hussain A, Graner M, Frederick B, Agarwal R and Deep G: Exosomes secreted under hypoxia enhance invasiveness and stemness of prostate cancer cells by targeting adherens junction molecules. Mol Carcinog 54: 554-565, 2015.

3 Li W, Li C, Zhou T, Liu X, Li X and Chen D: Role of exosomal proteins in cancer diagnosis. Mol Cancer 16: 145, 2017.

4 Endzeliṇš E, Berger A, Melne V, Bajo-Santos C, Soboḷevska K, Ābols A, Rodriguez M, Šantare D, Rudņickiha A, Lietuvietis V, Llorente A and Linē A: Detection of circulating miRNAs: comparative analysis of extracellular vesicle-incorporated miRNAs and cell-free miRNAs in whole plasma of prostate cancer patients. BMC Cancer 17: 730, 2017.

5 Rodríguez M, Bajo-Santos C, Hessvik NP, Lorenz S, Fromm B, Berge V, Sandvig K, Linē A and Llorente A: Identification of non-invasive miRNAs biomarkers for prostate cancer by deep sequencing analysis of urinary exosomes. Mol Cancer 16: 156, 2017.

6 Belov L, Matic KJ, Hallal S, Best OG, Mulligan SP and Christopherson RI: Extensive surface protein profiles of extracellular vesicles from cancer cells may provide diagnostic signatures from blood samples. J Extracell Vesicles 5: 25355, 2016.

7 Belov L, Hallal S, Matic K, Zhou J, Wissmueller S, Ahmed N, Tanjil S, Mulligan SP, Best OG, Simpson RJ and Christopherson RI: Surface profiling of extracellular vesicles from plasma or ascites fluid using DotScan antibody microarrays. Methods Mol Biol 1619: 263-301, 2017.

8 Endzeliņ̌s E, Melne V, Kalniņa Z, Lietuvietis V, Riekstiṇa U, Llorente A and Linē A: Diagnostic, prognostic and predictive value of cell-free miRNAs in prostate cancer: a systematic review. Mol Cancer 15: 41, 2016.

9 Kreimer S, Belov AM, Ghiran I, Murthy SK, Frank DA and Ivanov AR: Mass-spectrometry-based molecular characterization of extracellular vesicles: lipidomics and proteomics. J Proteome Res 14: 2367-2384, 2015.

10 Hurwitz SN, Rider MA, Bundy JL, Liu X, Singh RK and Meckes DG: Proteomic profiling of NCI-60 extracellular vesicles uncovers common protein cargo and cancer typespecific biomarkers. Oncotarget 7: 86999-87015, 2016. 
11 Cvjetkovic A, Jang SC, Konečná B, Höög JL, Sihlbom C, Lässer $\mathrm{C}$ and Lötval J: Detailed analysis of protein topology of extracellular vesicles-evidence of unconventional membrane protein orientation. Sci Rep 6: 36338, 2016.

12 Nakurte I, Jekabsons K, Zandberga E, Abols A, Linē A and Muceniece R: Proteome analysis of colorectal cancer cell line SW480 released extracellular vesicles. Key Engineering Materials 762: 3-7, 2018.

13 Bao B, Ali S, Ahmad A, Azmi AS, Li Y, Banerjee S, Kong D, Sethi S, Aboukameel A, Padhye SB and Sarkar FH: Hypoxiainduced aggressiveness of pancreatic cancer cells is due to increased expression of VEGF, IL-6 and miR-21, which can be attenuated by CDF treatment. PLoS One 7: e50165, 2012.

14 Schwanhäusser B, Busse D, Li N, Dittmar G, Schuchhardt J, Wolf J, Chen W and Selbach M: Global quantification of mammalian gene expression control. Nature 473: 337-342, 2011.

15 Sadovska L, Eglitis J and Line A: Extracellular vesicles as biomarkers and therapeutic targets in breast cancer. Anticancer Res 35(12): 6379-6390, 2015

16 Endzeliňš E, Ābols A, Bušs A, Zandberga E, Palviainen M, Siljander $\mathrm{P}$ and Linē A: Extracellular vesicles derived from hypoxic colorectal cancer cells confer metastatic phenotype to non-metastatic cancer cells. Anticancer Res 38(9): 5139-5147, 2018.

17 Lötvall J, Hill AF, Hochberg F, Buzás EI, Di Vizio D, Gardiner C, Gho YS, Kurochkin IV, Mathivanan S, Quesenberry P, Sahoo $\mathrm{S}$, Tahara H, Wauben MH, Witwer KW and Théry C: Minimal experimental requirements for definition of extracellular vesicles and their functions: a position statement from the International Society for Extracellular Vesicles. J Extracell Vesicles 3: 26913, 2014.

18 Thomas SN, Liao Z, Clark D, Chen Y, Samadani R, Mao L, Ann DK, Baulch JE, Shapiro P and Yang AJ: Exosomal proteome profiling: a potential multi-marker cellular phenotyping tool to characterize hypoxia-induced radiation resistance in breast cancer. Proteomes 1: 87-108, 2013.
19 Ji H, Greening DW, Barnes TW, Lim JW, Tauro BJ, Rai A, Xu R, Adda C, Mathivanan S, Zhao W, Xue Y, Xu T and Zhu HJ: Proteome profiling of exosomes derived from human primary and metastatic colorectal cancer cells reveal differential expression of key metastatic factors and signal transduction components. Proteomics 13: 1672-1686, 2013.

20 Zhou J, Li XL, Chen ZR and Chng WJ: Tumor-derived exosomes in colorectal cancer progression and their clinical applications. Oncotarget 8: 100781-100790, 2017.

21 Furuta K, Ikeda M, Nakayama Y, Nakamura K, Tanaka M, Hamasaki N, Himeno M, Hamilton SR and August JT: Expression of lysosome-associated membrane proteins in human colorectal neoplasms and inflammatory diseases. Am J Pathol 159: 449-455, 2001

22 Jensen SS, Aaberg-Jessen C, Christensen KG and Kristensen B: Expression of the lysosomal-associated membrane protein-1 (LAMP-1) in astrocytomas. Int J Clin Exp Pathol 6: 1294-1305, 2013.

$23 \mathrm{Xu} \mathrm{R}$, David W, Greening DW, Rai A, Ji H and Simpson RJ: Highly-purified exosomes and shed microvesicles isolated from the human colon cancer cell line LIM1863 by sequential centrifugal ultrafiltration are biochemically and functionally distinct. Methods 87: 11-25, 2015.

24 Chun S, Ahn S, Yeom CH and Park S: Exosome proteome of U87MG glioblastoma cells. Biology 5: 50, 2016.

25 Wang JL, Qi Z, Li YH, Zhao HM, Chen YG and Fu W: TGF $\beta$ induced factor homeobox 1 promotes colorectal cancer development through activating $\mathrm{Wnt} / \beta$-catenin signalling. Oncotarget 8: 70214-70225, 2017.
Received September 13, 2018

Revised October 1, 2018

Accepted October 2, 2018 